\title{
Histopathologic analysis of gills of Solea solea and Solea senegalensis wild marine and aquaculture populations
}

\author{
P. R. Werneck*, N. M. M. Lopes*, K. R. De Moraes*, A. Faustino** and F. Morgado* \\ * CESAM \& Departamento de Biologia, Universidade de Aveiro, Campus Universitário de Santiago, \\ 3810-193 Aveiro, Portugal \\ ** Instituto de Ciências Biomédicas Abel Salazar, Universidade do Porto, Portugal
}

The physiological condition of Solea solea and Solea senegalensis, caught in the natural environment and in aquaculture fish farms respectively, was determined by analyzing the histological changes occurred in the gills of the animals [1,2]. These two species were used because both occupy the same ecological niche but only Solea senegalensis is reared in Portuguese aquaculture farms. The analysis was performed using 19 specimens in total from both environments collected between January and March of 2011 in two different locations of the Portuguese coast (Leixões and Aveiro, Portugal). The lesions found in the gills samples corresponded to four types of fusions: basal fusion corresponding to fusions at the base of the secondary lamella; medium fusion corresponding to fusions up to half of the secondary lamella; total fusion corresponding to fusions in the total length of the lamella; apical fusion corresponding to the complete fusion of the entire lamella. Another form of lesion found were lamellar edema which consisted of two different type: apical edema corresponding to a swelling at the apex of the secondary lamella; lamellar edema corresponding to a swelling in the full length of the tissue of the secondary lamella. The last physiological modification found was the alteration of the quantity of mucous cells found and also of mucous released in the primary and secondary lamellae of the gills. The results revealed that the most commonly type of lesions found were: basal fusion (Fig. 1. C); medium fusion (Figure $1 \mathrm{~B}$ ); apical fusion (Figure $1 \mathrm{~A}$ ); total fusion (Figure $1 \mathrm{D}$ ); lamellar edema (Figure $1 \mathrm{E}$ ); apical edema (Figure $1 \mathrm{~F}$ ) and mucous cells disorganization in the gills (Figure $1 \mathrm{G}$ ). These data are very similar to other previously reported results in histopathologic studies $[1,2,3]$, and are most likely to result from: contamination with heavy metals; organic or pesticides contamination; contamination by hydrocarbons (oils, fuels; rapid changes in the temperature of the water; the time of recovery of the fishing nets in the case of Solea solea; contamination of the ice used in the storage process of Solea solea. The histological analyses are very useful to evaluate the physiological condition of the fish. The histological analyses are a very important tool which enables the rapid development of processes and supports sound ecological management decisions in order to protect and/or recover habitats and ecosystems in which these animals live, therefore, improving their physiological condition with obvious benefits as to the human consumer is concerned. The novelty in this study was the use of bottom duelling fish and the comparison established between wild specimens and aquaculture reared specimens. The final conclusion is that several types of contamination occurred both in the natural environment as in the aquaculture fish farms, resulting in the different types of lesions and physiological changes observed during our experiment.

\section{References}

1. Hibiya T. and Takashima F., Atlas of Fish Histology - Normal and Pathological Features. Kodansha, Tokyo, Japan, 1995

2. Malins D.C. and Ostrander G. K., Aquatic Toxicology - Molecular, Biochemical and Cellular Perspectives. Boca Raton, Florida USA, 2000

3. Sensini C. et al., First observations of histopathologic effects of 2,4,6-trinitrotoluene (TNT) in gills of European eel Anguilla anguilla (Linnaeus, 1758). Cell Biol. Toxicol. Siena, Italy, 2008 

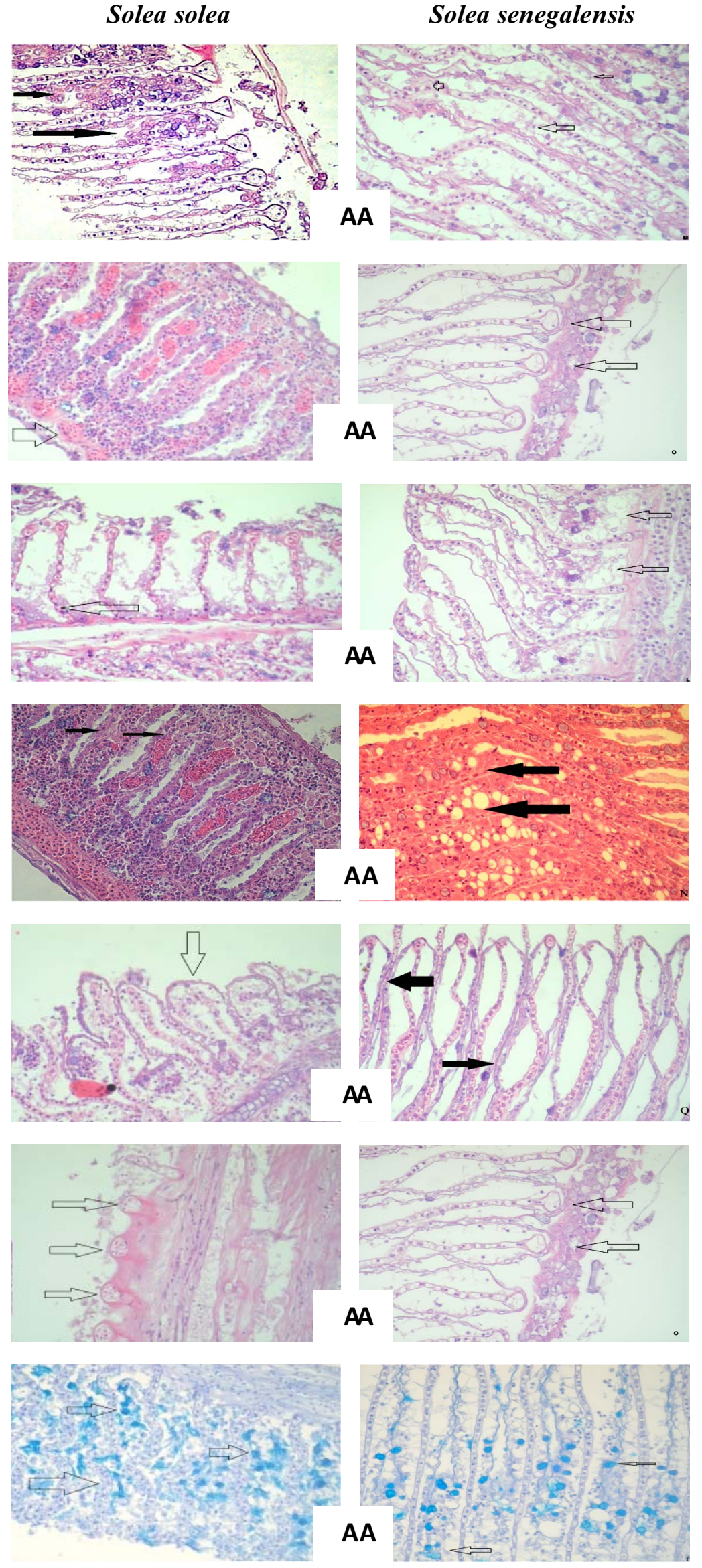

Figure 1. Histochemical analysis of Solea solea (left microphotographs) gills from natural coastal environment and Solea senegalensis (right microphotographs) gills from aquaculture fish farms. A: Arrows indicate the presence of Medial Fusion lesions (H\&E; 10x). B: Arrows indicates the presence of Apical Fusion lesions (H\&E); 40x). C: Arrows indicate the presence of Basal Fusion lesions. (H\&E; 40x). D: Arrows indicate the presence of Total Fusion lesions (H\&E, 10x). E: Arrows indicate the presence of Edema Lamellar lesions (H\&E; 40x). F: Arrows indicate the presence of Apical Edema lesions. (H\&E; 40x). G: Arrows indicate a high number of disorganized mucous cells and mucus (PAS and Alcian Blue, 40x). 